\title{
Determination of suspension viscosity from the flow velocity profile measured by Doppler Optical Coherence Tomography
}

\author{
J. Lauri, ${ }^{*}$ A. V. Bykov, and R. Myllylä \\ Optoelectronics and Measurement Techniques Laboratory, University of Oulu, P.O. Box 4500, 90014 \\ University of Oulu, Finland
}

Received May 15, 2011; accepted June 03, 2011; published June 30, 2011

\begin{abstract}
In this paper we present, for the first time to our knowledge, the experimental results for determining suspension viscosity in a capillary type viscometer from flow velocity profiles measured by Doppler Optical Coherence Tomography. The suspension of $0.3 \mu \mathrm{m}$ polystyrene microspheres in a glycerol-water solution was used as a model fluid. The viscosity of the suspension was controlled by glycerol concentration. The shear rate and the shear stress at the capillary wall were measured at six flow rates. The corresponding viscosities were calculated. The comparison of the measured shear rates with the values calculated according to the flow rate of the syringe precision pump was performed. Additionally, the viscosities of the studied suspensions were measured by a rotational viscometer.
\end{abstract}

The knowledge of rheological properties of fluids (e.g. viscosity, plasticity, etc.) is important for many industrial processes and microfluidic systems. Usually, the rheological parameters of a fluid at different conditions and flow rates are measured offline by rheometers. The prior knowledge of viscosity, especially in the case of non-Newtonian fluids like polymer solutions [1], pastes [2], and pulp [3], is important for predicting the process parameters and optimization of the pumping rate for an efficient flow with minimal pressure loss. The most common types of viscometers are rotational and capillary viscometers. According to a theoretical description, the viscosity of a fluid could be expressed as a function of the flow velocity profile in the capillary type viscometers. For Newtonian fluids in the static Poiseuille flow inside a capillary tube, the flow velocity profile can be calculated from the flow rate. However, fluids often show non-Newtonian behavior like shear thinning or wall slip. These effects cause errors in absolute viscosity measurements, thus the measured value is called apparent viscosity. There are different correction methods which take into account non-Newtonian behavior. However, they often require additional measurements which make the measurement process of non-Newtonian fluids slower and more laborious. Direct measurement of the flow velocity profile can make non-Newtonian correction methods unnecessary.

There are various methods for measuring the flow velocity profile: micro Particle Imaging Velocimetry ( $\mu$ PIV) [4], Laser Doppler Velocitymetry (LDV) [5-6], Ultrasound Velocity Profiling (UVP) [7-8]. However, these techniques are not free from significant disadvantages, e.g. limitations at high flow velocities, requirement for a transparent fluid and a poor spatial resolution. These limitations can be overcome by Doppler Optical Coherence Tomography (DOCT), which is a functional extension of Optical Coherence Tomography (OCT). DOCT [9] is a well-established high resolution imaging modality capable of measuring flow velocity profiles of highly scattering suspensions at micrometer scale. DOCT is commonly used in biophotonics to measure blood flow [10-12]. This technique is based on the detection of back scattered light and coherence gating. In this case, the indepth resolution is determined by the coherence length of the light source which can be very low (in the scale of several microns). The best in-depth resolution is achieved with utilizing a femtosecond Ti-Sapphire laser and a photonic crystal fiber for broad band light generation providing submicron resolution in depth [13].

Current studies of rheological parameters of suspensions, utilizing DOCT, have been insignificant apart from the latest study [14]. In this study we utilize DOCT technique, for the first time to our knowledge, in a pressure difference capillary viscometer to measure the flow velocity profile from which shear rate and viscosity can be determined. Also the nonNewtonian behavior of a fluid, e.g. wall slip in the range of DOCT resolution or shear thinning can be studied. In this study we implement the laboratory-built time domain DOCT setup [15] for the measurement of flow velocity profiles of polystryrene microspheres suspension in the controlled shear rate capillary viscometer.

The central wavelength of the device is $840 \mathrm{~nm}$ with a spectral band of 50nm, resulting in an axial resolution of $6 \mu \mathrm{m}$ in the air. The shear rates are estimated from measured flow velocity profiles and compared to the shear rates calculated from the flow rates of a syringe precision pump. Further, the pressure drop in the capillary is measured and viscosity is estimated. In addition, for reference, the suspensions viscosity is measured using a Brookfield DV-II rotational viscometer.

The velocity profile $v(r)$ contains a radial distribution of velocities thus the spatial shear rate $\dot{\gamma}$ can be calculated according to the following equation:

*E-mail: janne.lauri@ee.oulu.fi 


$$
\dot{\gamma}=-\frac{d v(r)}{d r}
$$

The shear stress $\tau_{\text {wall }}$ is calculated at the capillary wall by using the following equation:

$$
\tau_{\text {wall }}=\frac{\Delta P r}{2 L}
$$

where $\Delta P$ is the pressure drop along the capillary, $r$ is the radius and $L$ is the length of the capillary. The viscosity $\eta$ is defined by the ratio of shear stress and shear rate:

$$
\eta=\frac{\tau_{\text {wall }}}{\dot{\gamma}_{\text {wall }}}
$$

Velocity profiles are measured at the center of a round glass capillary with an inner diameter of $1 \mathrm{~mm}$, which is often used in capillary rheometers. The angle between the probing beam and the capillary surface is set to 69.5 degrees resulting in the measurement speed range of $1-120 \mathrm{~mm} / \mathrm{s}$. The used sampling frequency is of $200 \mathrm{kHz}$. Flow velocity profiles inside a capillary are calculated by using the following equation:

$$
v=\frac{f_{D} \lambda_{0}}{2 n_{m} \cos \theta_{m}}
$$

where $f_{D}$ is the measured Doppler frequency shift, $\lambda_{0}$ is the center wavelength of the light source, $n_{m}$ is the refractive index of the medium and $\theta_{m}$ is the Doppler angle inside the medium.

The pressure drop along the glass capillary is measured with a differential pressure sensor. The pressure sensor is connected to the glass capillary with a polymer T-connector with the inner diameter of $0.8 \mathrm{~mm}$. The other input of the pressure sensor is left free in air as well as the capillary outlet. The total length of the capillary in which the pressure drop is measured is $269 \mathrm{~mm}$, including the $19 \mathrm{~mm}$ part of the $0.8 \mathrm{~mm}$ T-connector. The effects of entrance and exit to the pressure drop are neglected because of the large lengthdiameter ratio. Pressure values are obtained during the flow velocity profile measurements. All the measurements are performed at the ambient temperature of $24.0 \pm 0.4^{\circ} \mathrm{C}$.

Glycerol-water solutions are prepared from $99.5 \%$ glycerol and distilled water with varying concentration ratio 50/50, $40 / 60,30 / 70$ and 20/80, respectively. Polystyrene particles of $0.3 \mu \mathrm{m}$ in $10 \% \mathrm{wt} / \mathrm{wt}$ suspension are added as scattering particles. The total concentration of particles was less than $0.17 \%$ in the final suspension. The concentration of polystyrene particles is adjusted, depending on the glycerol concentration to keep the scattering coefficient $\mu_{\mathrm{s}}$ constant to maintain the stable signal to noise ratio for different samples. The $\mu_{\mathrm{s}}$ was calculated to be $2.5 \mathrm{~mm}^{-1}$. The constant scattering coefficient among the different suspensions was verified from the attenuation slope of the DOCT signal envelope. The suspensions were sonicated for $30 \mathrm{~min}$ before the measurements to make particles evenly dispersed. The Reynolds number was calculated to be below 50, which indicated laminar flow.
Flow velocity profiles were measured through a $1 \mathrm{~mm}$ glass capillary and were fitted with parabola excluding flow rates of $150 \mathrm{ml} / \mathrm{h}$ and $200 \mathrm{ml} / \mathrm{h}$, in which the velocities in the center of the capillary exceeded the Nyquist frequency at the 200$\mathrm{kHz}$ sampling frequency. In this case only a part of the velocity profile was used on fitting. Figure 1(a) shows the average of the 5 measured flow velocity profiles at $200 \mathrm{ml} / \mathrm{h}$, $100 \mathrm{ml} / \mathrm{h}$ and $50 \mathrm{ml} / \mathrm{h}$. The velocity profile at $200 \mathrm{ml} / \mathrm{h}$ could be measured only at a distance of less than $300 \mu \mathrm{m}$ from the capillary wall because of the large Doppler angle and the used sampling frequency. The small sinusoidal oscillation, best seen at the flow rate $50 \mathrm{ml} / \mathrm{h}$, is a Doppler noise from the scanning piezostage. Figure 1(b) shows the close-up at the $100 \mu \mathrm{m}$ distance from the wall with error bars and fitted profiles (solid lines).

(a)

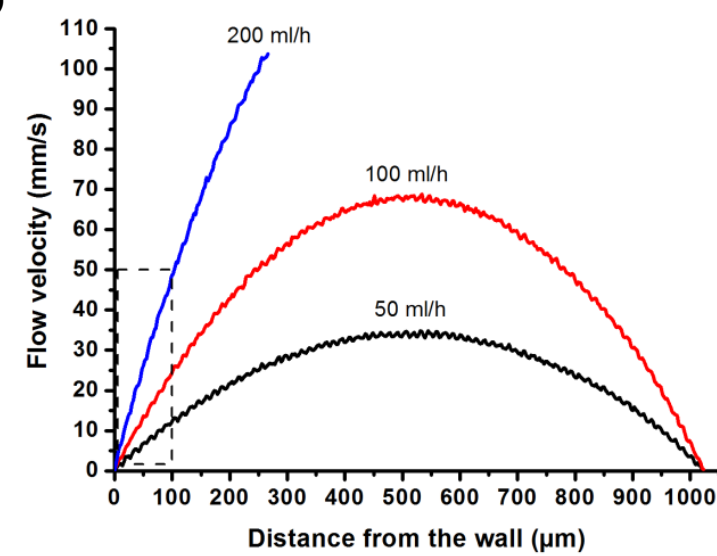

(b)

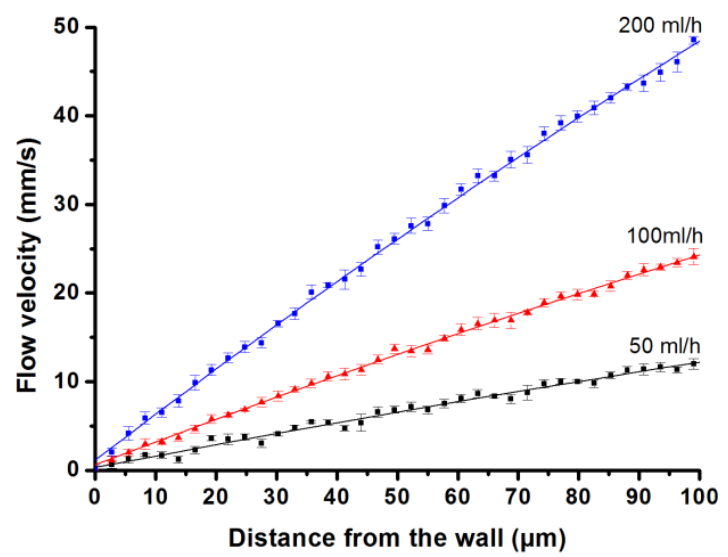

Fig. 1. Measured flow velocity profiles at flow rates of $200 \mathrm{ml} /, 100 \mathrm{ml} / \mathrm{h}$ and $50 \mathrm{ml} / \mathrm{h}$ (a). Profiles with parabolic fitting visualized at the capillary wall (b). The solid lines are fitted parabolas. 
The shear rates were calculated from the fitted velocity profiles at the wall. The results are shown in Table 1 together with the values calculated from the flow rate settings of the syringe pump. The estimated shear rates from the velocity profiles are in excellent agreement with the calculated values, the relative error is less than $1 \%$. The shear rate variation between samples is really consistent. Furthermore, by using the measured shear rates and pressure values, the viscosity was estimated with Eq. (3).

Table 1. Measured shear rates and theoretical shear rates with different flow rates. $\mathrm{G}$ is glycerol and $\mathrm{W}$ is water.

\begin{tabular}{c|c|c|c|c|c}
$\begin{array}{c}\text { Flow rate } \\
\mathrm{ml} / \mathrm{h}\end{array}$ & $\begin{array}{c}\text { Theoretical } \\
\text { shear rate }\end{array}$ & $\begin{array}{c}50 \% \mathrm{G} . \\
50 \% \mathrm{~W} .\end{array}$ & $\begin{array}{c}40 \% \mathrm{G} . \\
60 \% \mathrm{~W} .\end{array}$ & $\begin{array}{c}30 \% \mathrm{G} . \\
70 \% \mathrm{~W} .\end{array}$ & $\begin{array}{c}20 \% \mathrm{G} . \\
80 \% \mathrm{~W} .\end{array}$ \\
\hline 50 & 132.0 & 130.1 & 129.6 & 130.3 & 129.0 \\
75 & 198.0 & 195.0 & 195.7 & 195.5 & 193.6 \\
100 & 264.0 & 260.1 & 260.6 & 259.4 & 258.4 \\
125 & 330.0 & 324.8 & 324.6 & 324.1 & 322.2 \\
150 & 396.0 & 390.3 & 394.9 & 390.3 & 391.9 \\
200 & 528.0 & 524.6 & 521.9 & 527.5 & 537.9
\end{tabular}

The estimated viscosities are shown in Fig. 2 with solid symbols. In addition, the reference measurements of the rotational viscometer are shown with open symbols. The viscosities determined from the flow velocity profiles measured by DOCT show Newtonian behavior of the glycerol-water mixture although there is some increase in viscosity at a shear rate of $130 \mathrm{~s}^{-1}$. Unfortunately, the shear rate range of the used rotational viscometer ended where the capillary viscometer range started. There is a small difference between the viscometers at $130 \mathrm{~s}^{-1}$ but at higher shear rates the capillary viscometer value approaches the same value as the rotational viscometer. The differences of the viscosity values could be corrected by calibration of the rotational viscometer.

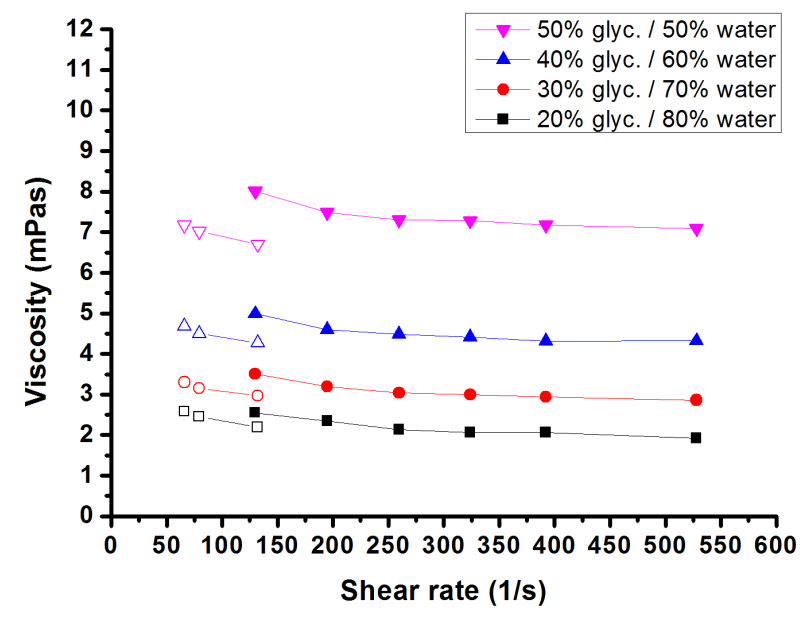

Fig. 2. Estimated viscosities measured from flow velocity profiles (solid symbols) and the corresponding viscosities measured with the rotational viscometer (open symbols).
In conclusion, we demonstraded a new type of device, utilizing the DOCT setup in a capillary viscometer design for direct flow velocity profile measurement. The shear rates were determined with high accuracy and the pressure drops along the capillary were measured. Absolute viscosities were calculated according to Eq. (3) and compared with the values measured by the rotational viscometer. One of the limitations of the DOCT technique is the requirement of a scattering solution to enable backscattering.measurement. Another limitation is related with spatial resolution: velocity measurements closer than $\sim 1-2 \mu \mathrm{m}$ from the wall are not possible. All the measured solutions showed Newtonian behaviour although more interesting is to measure nonNewtonian suspensions which undergo wall slip or have shear thinning behaviour. The DOCT method can be easily applied to slit rheometers where LDV already has been used. DOCT may be utilized to correct measurement errors caused by frictional heating, which produces large temperature gradients inside capillary rheometers operating at high shear rates $\left(>10^{6} \mathrm{~s}^{-1}\right)$. In addition, the DOCT method enables the use of the envelope signal for studying particle density inside the capillary or tube.

Authors acknowledge M. Sc. Markus Mikkola for his contribution for pressure measurement system design and Dr. Alexey Popov for calculations of the suspensions scattering coefficient.

\section{References}

[1] K.A. Hayes, M.R. buckley, I. Cohen, L.A. Archer, Phys. Rev. Lett. 101, 218301 (2008).

[2] N. Willenbacher, H. Hanciogullari, H.G. Wagner, Chem. Eng. Technol. 20, 557 (1997).

[3] M. Iotti, Ø.W. Gregersen, S. Moe, M. Lenes, J. Polym. Environ. 19, 137 (2011).

[4] M.T. Roberts, A. Mohraz, K.T. Christensen, J.A. Lewis, Langmuir 23, 8726 (2007).

[5] M. Schmidt, E. Wassner, H. Münstedt, Mech. Time-Depend. Mater. 3, 371, (1999).

[6] S. Schuberth, H. Münstedt, Rheol. Acta 47, 111 (2008).

[7] Th. Wunderlich, P.O. Brunn, Flow Meas. Instrum. 11, 63 (2000).

[8] J. Wiklund, I. Shahram, M. Stading, Chem. Eng. Sci. 62, 4277 (2007).

[9] S. Yazdanfar, M.D. Kulkarni, J.A. Izatt, Opt. Express 1(13), 424 (1997).

[10] Y. Zhao et al., Opt. Lett. 25(2), 114 (2000).

[11] J. Moger, S.J. Matcher C.P. Winlove, A. Shore, J. Biomed. Opt. 9(5), 982 (2004).

[12] S. Tamborski et al., Phot. Lett. Poland 1(2), 49 (2009).

[13] B. Povazay et al., Opt. Lett. 27(20), 1800 (2002).

[14] M. Harvey, T.A. Waigh, Phys. Rev. E 83, 031502 (2011).

[15] J. Lauri, M. Wang, M. Kinnunen, R. Myllylä, Proc. SPIE 6863, 68630F (2008) 